\title{
Orientational order and dynamics of the dendritic liquid crystal organo-siloxane tetrapodes determined using dielectric spectroscopy
}

\author{
K. Merkel, ${ }^{1,2}$ A. Kocot, ${ }^{1,2}$ and J. K. Vij ${ }^{1, *}$ \\ ${ }^{1}$ Laboratory of Advanced Materials, Department of Electronic Engineering, Trinity College, University of Dublin, Dublin 2, Ireland \\ ${ }^{2}$ Institute of Physics, University of Silesia, Katowice, Poland \\ G. H. Mehl and T. Meyer \\ Department of Chemistry, University of Hull, Cottingham Road, Hull HU6 7RX, United Kingdom \\ (Received 17 June 2005; revised manuscript received 8 December 2005; published 3 May 2006)
}

\begin{abstract}
The dielectric measurements have been carried out on the two zeroth generation dendrimers with four branched arms (called tetrapodes) based on the siloxane cores. The results are analyzed in the framework of the molecular theory of dielectric permittivity by Maier and Meier for nematogens. At least four molecular processes are resolved in the dielectric relaxation spectra in the nematic phase for each of the two tetrapodes. Three of them are assigned to the reorientation of the monomeric unit whereas the fourth is assigned to the rotation of the molecular segments in the individual arms of the monomeric unit around the long molecular axis. The dielectric relaxation strength of the low frequency process has been used to calculate the orientational order parameter. The dynamics of the resolved processes has been quantitatively analyzed using the results of the microscopic model of the rotational diffusion, given by Coffey and Kalmykov [W. T. Coffey and Yu. P. Kalmykov, Adv. Chem. Phys. 113, 487 (2000)] using the calculated order parameter. All molecular processes: the rotation around the short molecular axis (end-over-end rotation), precession around the director and the rotation around the long molecular axis (also called the spinning motion) are shown to have successfully been reproduced by the model. The anisotropy of the rotational diffusion coefficients gradually increases with a reduction in temperature, to a factor of 3 in the nematic phase relative to its isotropic phase.
\end{abstract}

DOI: 10.1103/PhysRevE.73.051702

\section{INTRODUCTION}

Currently, there is a growing interest in investigating systems with unusual molecular structure such as dendrimers [1-6] that exhibit mesomorphic properties. Dendrimers and structurally related multipodal systems called multipodes represent a class of macromolecules with a perfectly branched uniform structure in contrast to the hyperbranched polymers in which the branching occurs in a random fashion. Dendrimers have several generations, with a complete shell for each generation and this shell is branched out. The dendrimers are monodisperse, i.e., these have a narrow distribution of the molecular weights as opposed to the polymers. In some larger systems, the cooperative effects, due to crowding and/or nanosegregation (e.g., silicone/hydrocarbon or perfluorinated groups) involving the coupling of both the dendritic core and the branches containing mesogens are observed. In such systems, the overall shape and/or nanosegregation of the system determines the structure of the phase formed through self-assembly, and not by the properties of the individual mesogens [7-10]. The linkage between the mesogens and the dendritic cores is thus a crucial element for the phase formation. For systems below the limit of steric crowding at the periphery, strong decoupling (long alkyl spacers) leads to the behavior similar to that found for conventional side chain polymers. Nevertheless, for short spacers, the dendritic core and mesogens are typically strongly

\footnotetext{
*Author to whom correspondence should be addressed. Email address: jvij@tcd.ie
}

PACS number(s): 61.30.Gd, 42.70.Df, 77.80.-e, 78.30-j

coupled [8]. However, for most systems in the intermediate regime, the behavior is much more complex, and is dependent on the structure of the mesogen, the length, and the flexibility of the spacer (e.g., due to the presence of highly flexible siloxane groups). The mesogens normally have a tendency for parallel alignment, and the flexible spacers generally create disorder.

Oligomers consist of several monomeric groups connected covalently (generally 2-20), in a larger molecule. These can be of dendritic structure or alternatively may form a linear chain. It has been found that some oligomeric systems having mesogens and spacers form a layered structure $[1-4,11,12]$. In the low molar mass liquid crystals, however, it is now well known in liquid crystal physics that the order (or the alignment) is caused by the mesogens, whereas the long chains are responsible for disorder that makes the material to exhibit the liquid crystalline properties. In this context, multipodal or oligomeric systems placed in size in between the low molar mass compounds on the one hand and the large size dendrimers on the other extreme are particularly suitable candidates for a systematic investigation of the effects of attaching functional groups through flexible spacers to the dendritic core. In this paper, we are dealing with the zeroth generation dendrimer, which serves as the nucleus for the next generations of the dendrimers. Multipodes [13] in general, attached to the carbosilazane cores through flexible spacers, have recently been found to behave as though mesogens are decoupled more or less from the core [14]. In the system investigated here the synthesis of which will be described elsewhere [15], the compound has four branches and it is therefore called a tetrapode. Each arm contains the 
mesogen which on its own exhibits the nematic phase, and these arms are linked to the siloxane/silane core through the flexible spacers. The tetrapode is found to exhibit both the nematic and smectic phases [16].

Most applications of liquid crystals, whether low molar mass or polymeric, are based on using the anisotropic properties and their dependencies on pressure, temperature, and the external field. Basically, the macroscopic anisotropic properties of liquid crystals are governed by their molecular behavior, the alignment, and the orientational order of the constituent molecules $[17,18]$. One of the interesting problems in liquid crystals physics that still remains to be solved is the determination of a quantitative relationship between the molecular and the macroscopic properties. We can express this relationship through the microscopic tensor order parameters provided the interactions among the molecules can be neglected. It is therefore pertinent that the various order parameters of a given liquid crystal in different phases be determined. Any anisotropic property such as the dielectric permittivity, refractive index, magnetic susceptibility, elastic modulus, viscosity, etc. can be determined if only a set of the order parameters and the corresponding molecular properties are known. For example in the problem under discussion: the molecular properties are the dipole moments and the polarizability, whereas the macroscopic property is the relative permittivity.

In this paper we use the dielectric spectroscopic technique to measure $\varepsilon^{\prime}$ and $\varepsilon^{\prime \prime}$ of the homogeneously aligned tetrapodes. It is found that the alignment of these tetrapodes, as seen for the liquid crystalline glass cells under the polarizing microscope, fabricated similarly to the brass dielectric cells both in terms of the alignment and the cell thickness, is generally excellent. This gives us a confidence in the results obtained from the dielectric spectroscopic technique for these tetrapodes. If the molecule under discussion is parallel to the plane of the cell, then only the fluctuation of the transverse component of its dipole moment can give rise to the dielectric signal in terms of the frequency dependence of the real and imaginary parts of the permittivity. However, even in a homogeneously aligned cell, all the molecules do not lie flat on the windows of the cell. These are ordered with respect to the director, which may lie on the window for perfectly ordered sample or be tilted with respect to window plane for not so perfectly aligned sample. In either case, the molecules are somewhat tilted from the director. Therefore both components of the dipole moment parallel and perpendicular to the mesogens in principle can give rise to the dielectric modes in a single dielectric experiment on a homogeneously aligned sample. We find that these modes can be better resolved from each other if we are to differentiate the real part of permittivity with respect to log of frequency and analyze its frequency dependence than carrying out the analysis of the frequency dependence of $\varepsilon^{\prime \prime}$ alone. The latter includes the dc conductivity, whose effect is rather dominant at low frequencies and thus the low frequency modes cannot be easily determined from $\varepsilon^{\prime \prime}$ data alone. We shall later demonstrate that the new technique of analysis gives more reliable results for the temperature dependencies of the frequencies of the various modes. Several dielectric studies for low molar mass liquid crystals, dimers, and polymers [14,19-22] have already been reported in the literature. But even for the low molar mass liquid crystals, the data for the various relaxation modes is scarce [19] mainly because of the difficulty of carrying out the dielectric measurements at high frequencies on the aligned liquid crystalline samples. Nevertheless, even for dimers which involve rather complex structure of the mesogen, the measured dielectric spectrum is rather simple [20,21].

Dielectric relaxation for the system that consists of mesogens of the similar structure has already been studied for the dimers [21]. It was shown that the relaxation associated with the overall dipoles of the individual mesogenic cores are around and below the frequency of $10^{6} \mathrm{~Hz}$. Three different molecular modes were observed due to the overall dipolar reorientation. The fourth relaxation peak seen at higher frequencies was assigned to the rotation of the dipolar ester group around the long axes of the mesogen. In this case, however, though the mesogens of the tetrapodes are more or less decoupled from the core, there is still a finite restriction on the rotation of the mesogens resulting in higher viscosity of the material. Consequently the corresponding modes are shifted to the lower frequencies with respect to the case for the dimer.

\section{DIELECTRIC RELAXATION OF LIQUID CRYSTALS}

For uniaxial liquid crystals in the absence of measurable local biaxial order, the frequency dependence of the dielectric permittivity can be analyzed using Maier and Meier theory (M-M) [19,23]. Expressions for the real part of the permittivity parallel and perpendicular to the director, $\varepsilon_{\|}$and $\varepsilon_{\perp}$, are given as follows:

$$
\begin{gathered}
\varepsilon_{\|}(\omega)-n_{\|}^{2}=\frac{N h F^{2} g_{1}^{\|}}{3 \varepsilon_{0} k_{B} T}\left[\mu_{l}^{2}(1+2 S)+\mu_{t}^{2}(1-S)\right], \\
\varepsilon_{\perp}(\omega)-n_{\perp}^{2}=\frac{N h F^{2} g_{1}^{\perp}}{3 \varepsilon_{0} k_{B} T}\left[\mu_{l}^{2}(1-S)+\mu_{t}^{2}\left(1+\frac{1}{2} S\right)\right] .
\end{gathered}
$$

Here $\mu_{l}$ and $\mu_{t}$ are the longitudinal and transverse components of the molecular dipole moment to the molecular long axis. $N h F^{2} / 3 \varepsilon_{0} k_{B} T$ is the scaling factor for each dipolar contribution to the dielectric relaxation strength. $N$ is the number density of molecules, $\varepsilon_{0}$ is the permittivity of vacuum, $T$ is the absolute temperature, and $F$ and $h$ are the internal field factors for the reaction and the cavity fields, respectively. $g_{1}^{\perp}$ and $g_{1}^{\|}$are the anisotropic Kirkwood correlation factors defined for the director perpendicular and parallel to the electric field, respectively. For biaxial liquid crystalline phases, such as the biaxial nematic or $\mathrm{SmC}$ phases, it is possible to develop an equivalent set of equations by taking the phase and the molecular biaxialities into account [18]. However, the biaxial nematics need to be aligned along the two directors [24] and for alignment of only one of the directors, the material may behave more or less similarly to the uniaxial nematics especially for the homogeneous alignment. For the sake of convenience, the experiments in this paper are restricted to the planar homogeneous alignment and the major 


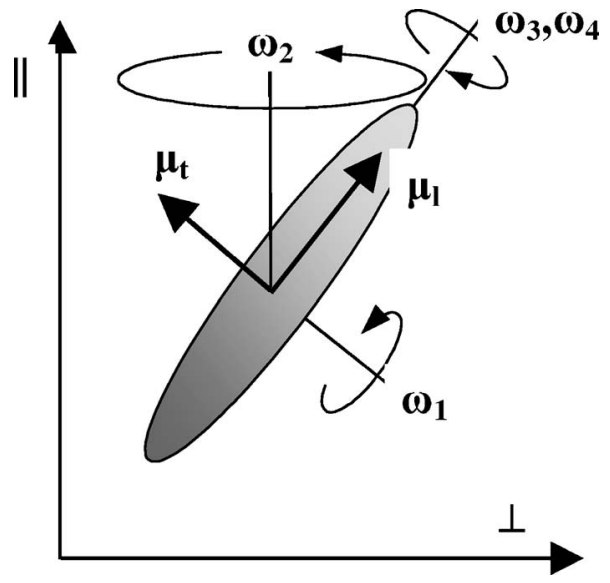

FIG. 1. Illustration of the mechanisms for the rotational relaxation modes for a molecule in the nematic potential. The parallel and perpendicular axes denote the direction of the electric field with respect to the director. The measurements are made with the electric field perpendicular to the director.

director is aligned along the window of the plane by rubbing of the alignment layer. Since the monomers in the tetrapodes may be tilted [16] with respect to the director, we may be able to observe dielectric modes for both $\varepsilon_{\perp}$ and $\varepsilon_{\|}$in a single dielectric experiment.

Each component of $\varepsilon(\omega)$, in Eqs. (1a) and (1b), contains two contributions from the total molecular dipole moment (Fig. 1) and each of these is expected to show at least two relaxation processes due to the different dependence of a component of the dipole moment to the two permittivities. The contributions to the transverse relative permittivity are usually not well separated from each other in the frequency spectrum. However, using our technique for the analysis of the data to be discussed later, we are able to separate the various processes more easily. The molecular dynamics can approximately be described in terms of the three rotational modes: (a) the end-over-end rotation, (b) the precessional motion of the long molecular axis around the director of the phase, and (c) the rotation about its own long molecular axis. So far it has been assumed that the molecules are rigid in that only a single dipole in each of the molecule contributes to the dielectric permittivity. However, if there are a number of dipolar groups present, which are not rigidly connected to each other, these then may make separate contributions to the total dielectric permittivity.

The frequency dependencies of the real and the imaginary parts of the permittivity allow the different contributions to be separated. The temperature dependence of the dielectric relaxation strength for each component is primarily determined by the variation of the principal order parameter $S$ with temperature. In order to obtain more information about the nature of the rotational dynamics in liquid crystals, it is necessary to employ suitable microscopic models [25-27]. Such models evaluate the time correlation function $\Phi_{m n}(t)$ of the fluctuating components of the molecular dipoles. It was shown numerically [25] and by analytical methods [26,27] that for the rotational diffusion model, the time dependence of $\Phi_{m n}(t)$ can sufficiently be described by a single correlation time for each component contributing to Eq. (2) given below. Here the subscripts $m$ and $n$ are the indices of the Wigner function. A relation can approximately be established between these times and the four different rotational modes: such that $\left(\omega_{1}\right)^{-1} \sim \tau_{00}, \quad\left(\omega_{2}\right)^{-1} \sim \tau_{10}, \quad\left(\omega_{3}\right)^{-1} \sim \tau_{11}, \quad$ and $\left(\omega_{4}\right)^{-1} \sim \tau_{01}$ [25], where $\tau_{00}, \tau_{10}, \tau_{11}$, and $\tau_{01}$ are the corresponding relaxation times.

The temperature dependence of the dielectric relaxation strengths associated with different modes in terms of the order parameter is given by a set of Eqs. (1a) and (1b). There is, however, no general theory as yet for the temperature dependence of the relaxation frequencies of liquid crystals associated with the different modes. For a nematic potential of the form [28] $U / k_{B} T=-\sigma \cos ^{2} \theta$, where $\theta$ is the angle between the axis of symmetry of the molecule and the $Z$ axis of the laboratory coordinate system. By assuming the rigid rodlike molecular structure, the approximate expressions for the reduced relaxation times (retardation factors) have been derived by Coffey and Kalmykov (C-K) [26,27] as given below

$$
\begin{gathered}
\frac{\tau_{00}}{\tau_{D}}=\left(\frac{e^{\sigma}-1}{\sigma}\right)\left(\frac{2 \sigma \sqrt{\sigma / \pi}}{1+\sigma}+2^{-\sigma}\right)^{-1}, \\
\frac{\tau_{01}}{\tau_{D}}=\frac{1-S}{1+\Delta(1-S)+\frac{1}{2} S}, \\
\frac{\tau_{10}}{\tau_{D}}=\frac{1-S}{1+\frac{1}{2} S}, \\
\frac{\tau_{11}}{\tau_{D}}=\frac{2+S}{2+\Delta(2+S)-\frac{1}{2} S} .
\end{gathered}
$$

$\sigma$ is the barrier height parameter and proportional to the uniaxial order parameter, $S, \tau_{D}$ is the Debye relaxation time for the isotropic phase, and $\Delta=\frac{1}{2}\left[D_{\|} / D_{\perp}-1\right]$ is a measure of the anisotropy of the rotational diffusion tensor. The parameter $\Delta$ can be calculated by fitting the ratio of relaxation times as in Eqs. (2b) and (2d) to the order parameter, $S$. This will be discussed later in Sec. IV C.

Recently molecular theory of dielectric relaxation for nematic dimers has been given by Stocchero et al. [29] to explain the unexpected and interesting results for the frequency dependence of the complex permittivity of dimers. In this paper we investigate the tetrapodes, which are even more complex than the dimers. Nevertheless we explain our results in terms of the simple Meier-Saupe potential model worked out more precisely by Coffey and Kalmykov [26,27].

\section{EXPERIMENT}

\section{A. Sample description, molecular structure, and the dipole moment}

Two organo-siloxane tetrapodes have experimentally been investigated. One of these tetrapodes involves an asymmetri- 
(a)

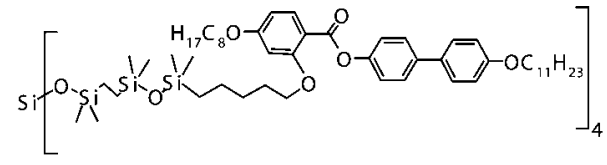

(b)

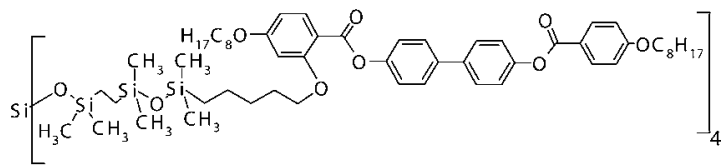

FIG. 2. (a) Structure of the tetrapode with asymmetric mesogenic group labeled as $A$. (b) Structure of the tetrapode with symmetrical mesogenic group labeled as $B$.

cal mesogen with three phenyl rings and different ester end groups; the molecular structure of this is shown in Fig. 2(a), hereinafter called $A$. The second involves a symmetrical mesogen with four phenyl groups with the same ester end groups and the molecular structure is shown in Fig. 2(b); hereinafter called tetrapode $B$. For both tetrapodes, the transition temperatures and the associated enthalpies have been determined for the second heating cycles using the differential scanning calorimetry (DSC) and the polarizing microscopy [13]. The structure of the monomers $A$ and $B$ have been optimized using the method of the density functional theory (DFT) including the hybrid B3LYP functional and standard polarized $6-31 \mathrm{G}^{*}$ basis set. The longitudinal and transverse components of the dipole moment of the monomer $A$ have been calculated to be 3.61 and $0.98 \mathrm{D}$, respectively. For monomer $B$, these are found to be 1.33 and $1.43 \mathrm{D}$, respectively, for the most probable conformation. These components give rise to a total dipole moment $\mu=3.74 \mathrm{D}$ and $\mu=1.95 \mathrm{D}$ for monomers $A$ and $B$.

\section{B. Sample preparation and the experimental details}

The sample cells for dielectric measurements have been prepared using gold-plated brass electrodes. For planar alignment of the sample, the conducting inner surfaces were spin coated with a $0.3 \%$ polyvinyl alcohol (PVA), alignment layer, and rubbed unidirectionally. Mylar foil was used as a spacer and two homogeneous cells were prepared. The thicknesses of the cells were determined from the measured capacitances of the empty cell and the cell gap attained in each of the cells was of the order of $12 \mu \mathrm{m}$. Cells were filled with the sample in its nematic phase at a temperature of approximately $5 \mathrm{~K}$ below its isotropization temperature.

The real and the imaginary parts of the relative dielectric permittivity $\varepsilon^{\prime}$ and $\varepsilon^{\prime \prime}$ (called dielectric permittivity from here afterwards) were measured over a frequency range $0.1 \mathrm{~Hz}-10 \mathrm{MHz}$ using a dielectric spectrometer (model Apha) manufactured by Novocontrol Ltd. The temperature accuracy of the dielectric cell was better than $\pm 0.1 \mathrm{~K}$. The ac measuring voltage applied to the sample was set at $100 \mathrm{mV}$. Dielectric measurements are extended for some cases to $30 \mathrm{MHz}$ using Solartron 1260A.

\section{RESULTS AND DISCUSSION}

The dielectric spectra of two tetrapodes in the planar homogeneous geometry are measured in the various phases. The result is shown in Fig. 3(a) for tetrapode $A$ and in Fig. 3(b) for tetrapode $B$ in temperature range of the nematic phase.

The dielectric spectra are fitted to Havriliak-Negami equation [30], which was applied to a set of $n$ relaxation processes

$$
\varepsilon^{*}(\omega)-\varepsilon_{\infty}=\sum_{j=1}^{n} \frac{\Delta \varepsilon_{j}}{\left[1+\left(i \omega \tau_{j}\right)^{\alpha}\right]^{\beta}} .
$$

In Eq. (3), $\varepsilon^{*}(\omega)$ is the frequency dependent complex permittivity, $\varepsilon_{\infty}$ is the high-frequency permittivity, $j$ is a variable denoting the number of relaxation processes up to $n, \tau_{j}$ is the relaxation time of the $j$ th relaxation process, $\alpha$ and $\beta$ are the fitting parameters of the symmetric and asymmetric relaxation processes, and $\Delta \varepsilon_{j}$ is the dielectric relaxation strength of the $j$ th process. For most temperatures at which the samples are investigated, the parameter $\beta$ was fixed to be unity for the entire range of temperatures. In such a case the relaxation time of a particular process is independent of $\alpha$ and is related to the relaxation frequency $f_{j}$ (or the angular frequency $\omega_{j}$ ) as follows: $\tau_{j}=1 / \omega_{j}=1 / 2 \pi f_{j}$. The dielectric relaxation times are determined for the homogeneously aligned tetrapode $A$ in the temperature range of the isotropic and nematic phases and for $B$ in the isotropic, nematic and its smectic phases. For a decomposition of the relaxation modes we have analyzed the derivative of the real part of the permittivity $d \varepsilon^{\prime} / d(\ln f)$.

$$
\begin{gathered}
\frac{d \varepsilon^{\prime}}{d \ln f}=\frac{d \varepsilon^{\prime}}{d \ln \omega}=\sum_{j=1}^{n} \operatorname{Re} \frac{\Delta \varepsilon_{j} \alpha\left(i \omega \tau_{j}\right)^{\alpha}}{\left[1+\left(i \omega \tau_{j}\right)^{\alpha}\right]^{2}}, \\
\frac{d \varepsilon^{\prime}}{d \ln \omega}=\sum_{j=1}^{n} \frac{\Delta \varepsilon_{j} \alpha_{j}\left(\omega \tau_{j}\right)^{\alpha_{j}}\left\{2\left(\omega \tau_{j}\right)^{\alpha_{j}}+\left[1+\left(\omega \tau_{j}\right)^{2 \alpha_{j}} \cos \frac{\pi \alpha_{j}}{2}\right] \cos \frac{\pi \alpha_{j}}{2}\right\}}{\left[1+\left(\omega \tau_{j}\right)^{2 \alpha_{j}}+2\left(\omega \tau_{j}\right)^{\alpha_{j}} \cos \frac{\pi \alpha_{j}}{2}\right]^{2}} .
\end{gathered}
$$



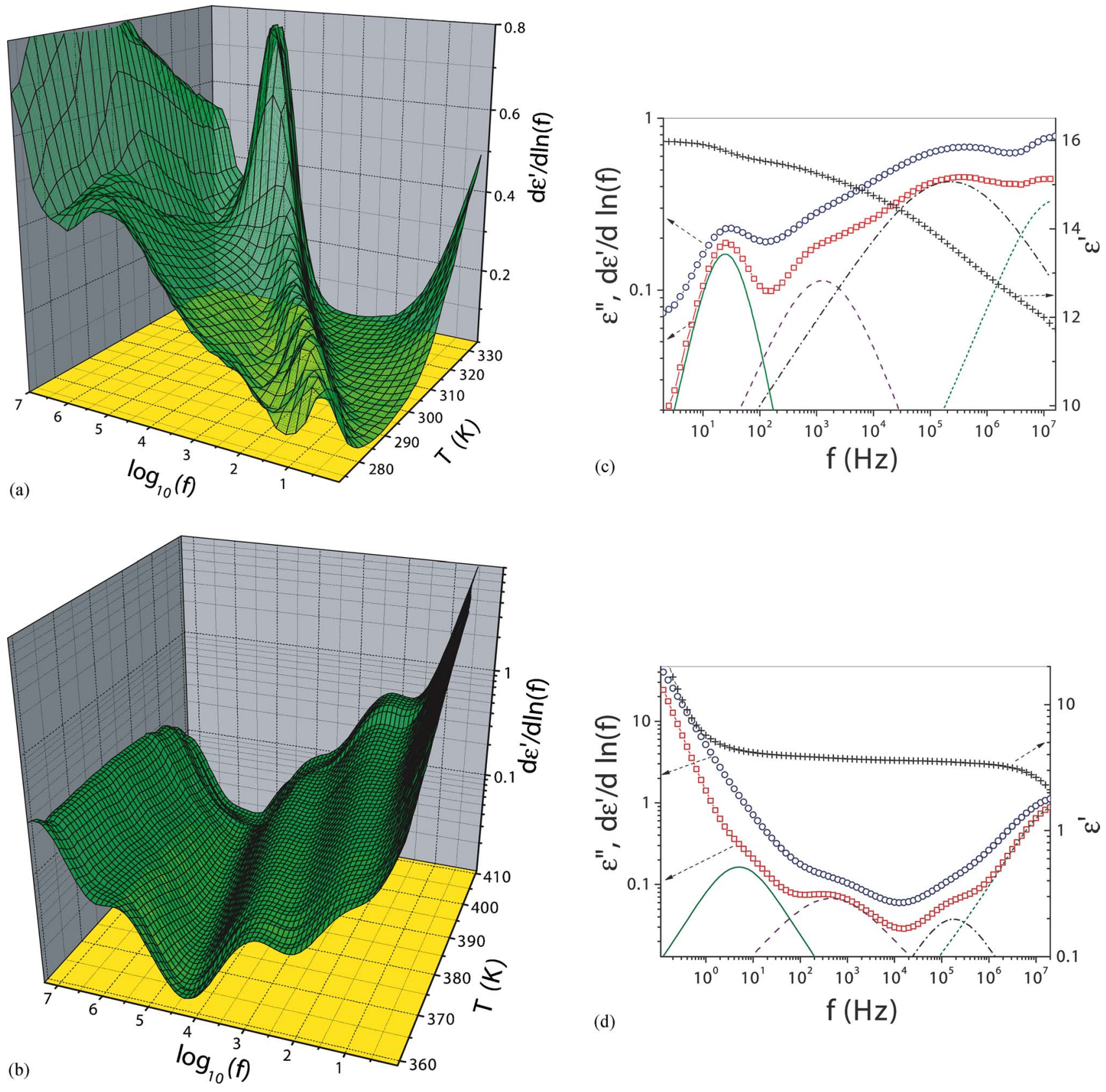

FIG. 3. (Color online) (a) Three-dimensional (3D) plot of the derivative of the relative dielectric permittivity with respect to (ln $f$ ) for tetrapode $A$ for different temperatures. (b) 3D plot of the derivative of the relative dielectric permittivity with respect to (ln $f$ ) for tetrapode $B$ for different temperatures. (c) Frequency plot of the relative dielectric permittivity for tetrapode $A$ at the temperature $285 \mathrm{~K}:+$ denotes $\varepsilon^{\prime}$, $\bigcirc$ denotes $\varepsilon^{\prime \prime}, \square$ denotes $d \varepsilon^{\prime} / d(\ln f)$, the derivative of $\varepsilon^{\prime}$ with respect to $(\ln f)$, full line, dashed line, dashed-dotted line, and short dashed lines are the deconvoluted components of $d \varepsilon^{\prime} / d(\ln f)$. (d) Frequency plot of the relative dielectric permittivity for symmetrical tetrapode $B$ at the temperature $365 \mathrm{~K}$ : + denotes $\varepsilon^{\prime}, \bigcirc$ denotes $\varepsilon^{\prime \prime}, \square$ denotes $d \varepsilon^{\prime} / d(\ln f)$ : the derivative of $\varepsilon^{\prime}$ with respect to (ln $f$ ), full line, dashed line, dashed-dotted line, and short dashed lines are the deconvoluted components of $d \varepsilon^{\prime} / d(\ln f)$.

For $\alpha=1, d \varepsilon^{\prime} / d(\ln \omega)$ is proportional to the square of $\varepsilon^{\prime \prime}$. The dielectric strength for each process $\Delta \varepsilon_{j}$ is also found from the fitting. We find that this method of analysis for determining the frequency of modes is more convenient than the analysis using the dielectric loss spectrum since it offers a better separation of the peaks. The peak positions lie at the same position as for $\varepsilon^{\prime \prime}$ as demonstrated here. For the Debye model of the dielectric relaxation, the $\varepsilon^{\prime \prime}$ peaks are 1.5 times wider (on the log-frequency scale) (half-width is $=1.14 \mathrm{de}$ - cades) than the corresponding peaks of $d \varepsilon^{\prime} / d(\ln f)$ (halfwidth $=0.75$ ). The overlapping of the peaks and a large ionic conductivity make the $\varepsilon^{\prime \prime}$ spectra rather difficult to deconvolute. A separation of peaks is clearly seen for $d \varepsilon^{\prime} / d(\ln f)$, see Figs. 3(c) and 3(d) for the two tetrapodes.

For the planar sample geometry in the nematic phase we measure the perpendicular $\varepsilon_{\perp}$ component of the dielectric permittivity. As stated in the Introduction, when the sample is macroscopically not perfectly aligned (the local axis of the 

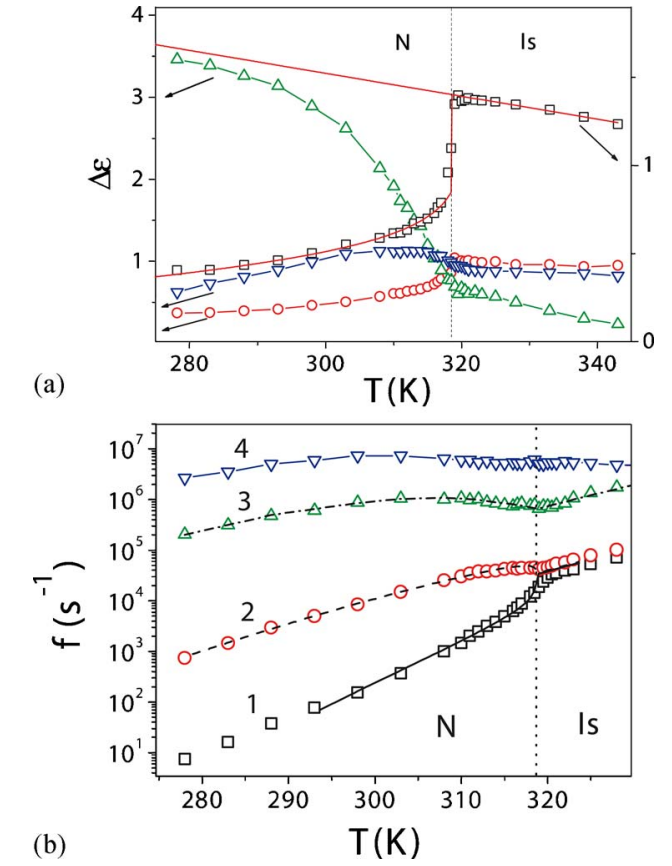

FIG. 4. (Color online) (a) Dielectric strength of the deconvoluted modes for tetrapode $A$ : $\square-\Delta \varepsilon_{1}, \bigcirc-\Delta \varepsilon_{2}, \triangle-\Delta \varepsilon_{3}, \nabla-\Delta \varepsilon_{4}$. Solid continuous line shows fitting of the M-M model to experimental data of the low frequency mode. (b) Relaxation frequencies of the deconvoluted modes for tetrapode $A$ : $\square-f_{1}, \bigcirc-f_{2}, \triangle-f_{3}$, $\nabla-f_{4}$. Solid and dashed lines show fitting of the Coffey-Kalmykov model to the experimental data.

nematic potential may fluctuate) or the director may make a finite pretilt angle with the substrate, then the relaxation process or the processes characteristic of the parallel component of the permittivity $\varepsilon_{\|}$may also be detected in the planar geometry (see Fig. 1). If the potential barrier is very high, then an additional high frequency non-Arrhenius mode may appear for the longitudinal component of the dipole due to the librational motion within the wells of the potential [31].

\section{A. Results for the tetrapode $A$ with the asymmetric core}

A frequency scan of the dielectric permittivity at room temperature for the tetrapodes, indicates contributions to it from up to four relaxation modes in the frequency range $1 \mathrm{~Hz}-10 \mathrm{MHz}$. The observed peaks are centered at frequencies of: $f_{1}=10^{2} \mathrm{~Hz}, f_{2}=10^{4} \mathrm{~Hz}$, and $f_{3}=5 \times 10^{5} \mathrm{~Hz}$. Mode 1 is assigned to be the end-over-end rotation, mode 2 is assigned to the precessional motion of the molecular directors about the phase director, and mode 3 is assigned to the molecular rotation about its own long molecular axis. Dielectric permittivity measured at the maximal frequency is found to be still reasonably high. In fact, the dielectric spectra, on the high frequency side, show the left wing of a rather strong peak with the maximum centered close to the high frequency limit of the experimental window. This implies that the additional process, $f_{4}$, or processes do contribute to the dielectric permittivity. Relaxation spectra of $d \varepsilon^{\prime} / d(\ln f)$ in the experimental frequency range of $1-10^{7} \mathrm{~Hz}$ are subsequently fitted to Eq. (4) for $n=4$ relaxation processes. Figures 4(a) and 4(b) show the dielectric relaxation strengths and the relaxation frequencies for the four separated relaxation processes, respectively.

One notes from Fig. 1 that the lowest frequency mode is contributed by the longitudinal, and the higher frequency mode $f_{3}$ is contributed by the transversal components of the dipole moment, respectively. Frequency of mode 2 (middle frequency one) is almost two orders of magnitude lower than that of mode 3 so the transversal component is already averaged in the time scale of mode 2. On approaching the $N-I$ phase transition, the two low frequency modes $f_{1}$ and $f_{2}$ merge into each other and a single relaxation process in the isotropic phase can describe this. Temperature dependences of the frequencies $f_{1}$ and $f_{2}$ of two modes clearly demonstrate that both of these modes are strongly affected by the orientational order. The third process, of the higher frequency, $f_{3}$, is considered to be the rotation of the transverse component of the dipole moment, $\mu_{t}$, around the long molecular axis. Similarly the fourth process, $f_{4}$, can be related to the rotation around the long molecular axis. However, relatively high frequency of this mode indicates that only the part of the mesogenic core can contribute to this motion (likely part with the dipolar ester group) and this is distinct from mode 3 , insofar its temperature dependence is rather weak. Peak frequencies, the dielectric strength of each mode, and the low frequency value of permittivity $\Delta \varepsilon_{\perp}-\varepsilon_{\infty \perp}$ are shown in Figs. 4(a) and 4(b).

According to the Maier-Meier theory [23], the dielectric strengths of the lowest or first mode in this case, $\Delta \varepsilon_{l}$, is proportional to the square of the longitudinal component of the dipole moment $\mu_{l}^{2}$ and should follow Eq. (1b). Hence, we are justified in using the expression

$$
\Delta \varepsilon_{l}=\frac{N h F^{2} g_{l}^{\perp} \mu_{l}^{2}}{3 \varepsilon_{0} k_{B} T}(1-S) .
$$

Using the results of the dielectric experiment, the scaling factor can be determined in the isotropic phase close to the $I-N$ transition when $S \cong 0$. As a first approximation, the Kirkwood correlation factor is assumed to be independent of temperature and its value is fixed to unity, we then calculate the dependence of $S$ on temperature. The result presented for $S$ in Fig. 5, is found to be in good accord with that found from the IR experiment. Temperature dependence of the dielectric relaxation strength can be reproduced satisfactorily using the order parameter obtained from the IR measurements [24], in the temperature range within the nematic phase. Both IR and dielectric results for $S$ can well be reproduced using Picken, Noriez, and Luckhurst approximation [32] of the MaierSaupe (M-S) theory [33]. The solid line in Fig. 5 shows the fit to the modified M-S model given by Chirtoc et al. [34], $S=S^{* *}+\left(1-S^{* *}\right)\left(1-T / T^{* *}\right)^{b}$, to the result obtained from dielectric experiments, where $T^{* *}$ is the effective second order (quasicritical or quasitricritical) phase transition temperature and $S^{* *}$ is finite at $T=T^{* *}$. The critical exponent $b$ is found to be 0.238 and $S^{* *}=0.085$. The value of the exponent is not much different from the tricritical behavior $(b=0.25)$ [17]. From Eq. (5) we can also calculate the longitudinal component of the dipole moment, $\mu_{l}=0.55 \mathrm{D}$, again by assuming 


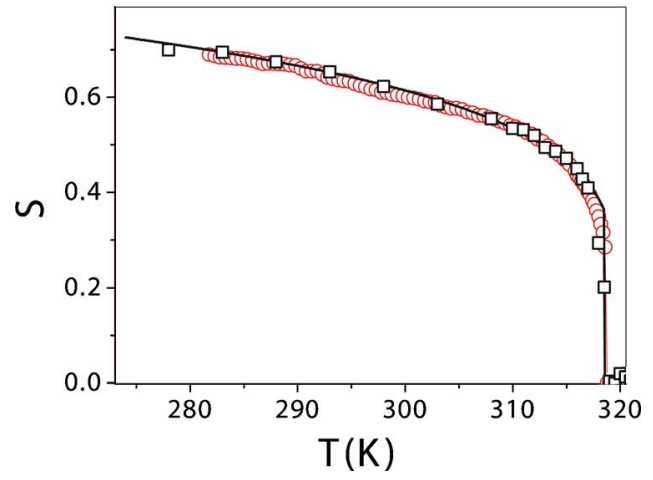

FIG. 5. (Color online) The orientational order parameters of tetrapode $A$ : calculated from the dielectric strength- $\square$, and from IR absorbance dichroism of phenyl stretching vibration- $\bigcirc$. Solid continuous line shows the fitting of the Maier-Saupe model to the dielectric data.

that in the vicinity of the isotropic phase $g \cong 1$. The dipole moments that contribute to the dielectric permittivity are located in the mesogenic core of the molecule and these are the dipole moment of the carboxyl group and three dipoles of the alkoxy group. Obviously, the magnitude of the total dipole moment depends on the conformation of the tetrapode branches themselves. For the most probable extended conformation of the monomeric part, it is reasonable to assume that alkoxy dipoles in the terminal chains can cancel with each other, so the net contributions in reality arise from the dipoles of the carboxyl and alkoxy groups in the ortho position. For such a configuration, the density functional theory simulation of the structure predicts $\mu_{l}=0.98 \mathrm{D}$ per monomeric unit. However, the experimental value of the overall dipole component is even lower than the simulated value because the components may partly cancel out with each other.

The third process is assigned to the rotation of the transverse component of the dipole moment around the long molecular axis using the relevant part of Eq. (1b), the dielectric strength of the third mode $\left(\Delta \varepsilon_{3}\right)$ is expected to satisfy the following equation:

$$
\Delta \varepsilon_{3}=\frac{N h F^{2} g_{1}^{\perp} \mu_{t}^{2}}{3 \varepsilon_{0} k_{B} T}\left(1+\frac{1}{2} S\right) .
$$

The observed temperature dependence of $\Delta \varepsilon_{3}$ [Fig. 4(a)] does not imply strong dependence of the Kirkwood correlation factor $g$. We can calculate the transverse component of the dipole moment, $\mu_{t}$, close to the $I-N$ transition temperature by assuming the Kirkwood correlation factor to be unity. We then find that $\mu_{t}$ is lower than predicted by the DFT simulated for one of the branches of the tetrapode. Furthermore, mode 3 is assigned to a rotation of each arm of the tetrapode independently of the other arms. The rotation of the entire molecule (which would normally occur at a much lower frequency) perhaps cannot be seen in the frequency window of our experiment. The rotation of each arm of the tetrapode as opposed to the entire tetrapode is justified in view of the fact that the spacers are flexible enough and consequently it is reasonable to expect that the reorientation

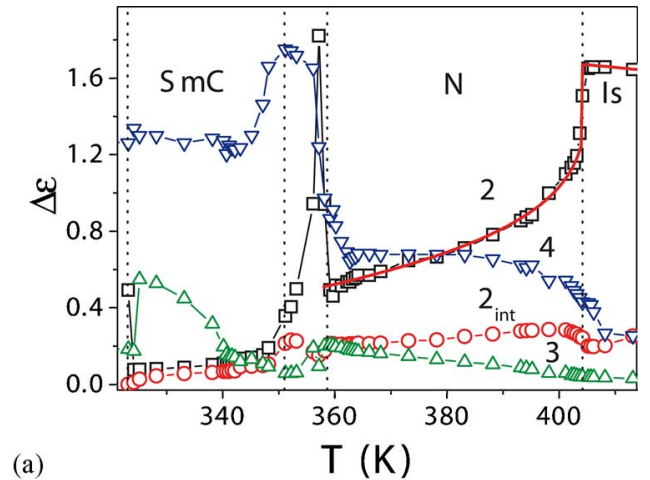

(a)

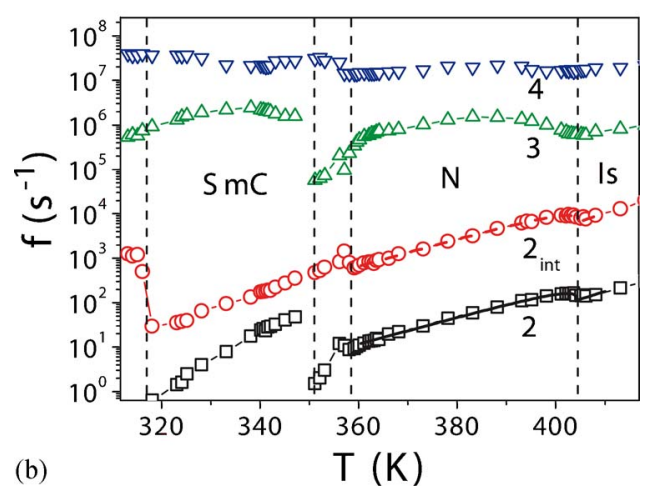

FIG. 6. (Color online) (a) Dielectric strength of the deconvoluted modes for tetrapode $B$ : $\square-\Delta \varepsilon_{2}, \bigcirc-\Delta \varepsilon_{2 \text { int }}, \Delta-\Delta \varepsilon_{3}, \nabla-\Delta \varepsilon_{4}$. Solid line shows fitting of the M-M model to the experimental data of the low frequency mode 2. (b) Relaxation frequencies of the deconvoluted modes for tetrapode $B: \square-f_{2}, \bigcirc-f_{2 \text { int }}, \triangle-f_{3}, \nabla-f_{4}$. Thick (solid and dashed) lines show the fitting of experimental data to the Coffey-Kalmykov model.

of the monomeric part is partly decoupled from the central part of the tetrapode.

\section{B. Results for tetrapode $B$ with the symmetric core}

For tetrapode $B$, up to four processes are detected in the temperature range of the nematic phase as for $A$. The dielectric strengths of four processes: mode 2, 2int, 3, and 4 are given in Figs. 6(a) and 6(b). We cannot investigate the dynamics of mode 1 (the end-over-end rotation for this tetrapode as this lies too close to the lowest frequency of the experimental window. The shift to lower frequency for this mode compared to that for tetrapode $A$ is possibly due to the longer length of the monomer involved. On the other hand, we can determine its relaxation strength by subtracting the relaxation strength of all other modes from the total permittivity. The low frequency mode seen in the dielectric spectrum lying in the frequency range $10-100 \mathrm{~Hz}$ is assigned to mode 2 (the precessional motion) due to the particular manner of its dependence of the peak frequency on temperature. We can also detect an additional higher frequency mode that corresponds to the precessional motion of the one half of the monomeric unit (not attached to the spacer), assigned as mode 2int (mode 2 internal). The process, of frequency $(\sim 1 \mathrm{MHz})$ is considered to be the rotation of the transverse component of the dipole moment, $\mu_{t}$, around the long mo- 


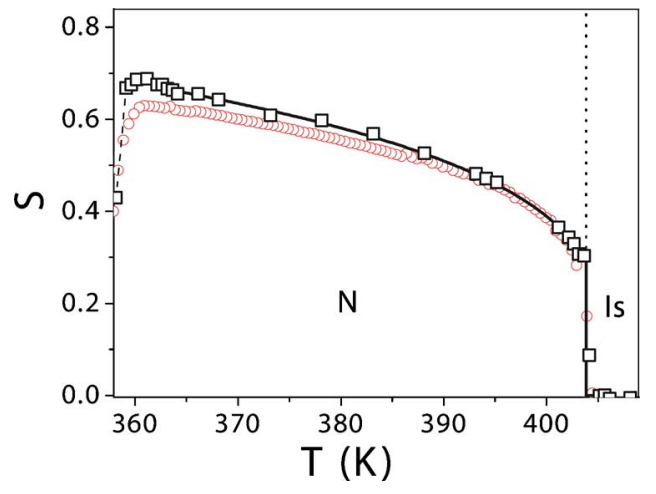

FIG. 7. (Color online) The orientational order parameters of the tetrapode $B$ : calculated from the dielectric strength- $\square$, and from IR absorbance dichroism of phenyl stretching vibration- $\bigcirc$. Solid line shows the fitting of the Maier-Saupe model to the dielectric data. The value of the exponent $=0.243$.

lecular axis and is assigned to mode 3 . The fourth process observed similarly to that in monomer $A$ is related to the rotation of the part of the mesogenic core around its long molecular axis. The dielectric strength of mode 1 is found to have similar temperature dependence to that of mode 2 and is not shown in Fig. 6(a). In a similar way as before, the temperature dependence of the dielectric relaxation strength of the low frequency mode can be well predicted using the $S$ parameter obtained from the IR experiment [24]. It is also possible to evaluate the order parameter from $\Delta \varepsilon_{l}$ using Eq. (5).

The temperature dependence of the order parameter for tetrapode $B$ is shown in Fig. 7. This is in quite good agreement with $S$ obtained from the IR experiment at the higher temperatures, but gradually exceeds IR result on lowering the temperature. This is probably due to the reason that the Kirkwood correlation factor may increase above unity on approaching the $\mathrm{N}-\mathrm{SmC}$ phase transition. The critical exponent for the temperature dependence of the order parameter for IR results is found to be 0.243 and $S^{* *}=0.09$. These are also not much different from the tricritical behavior as already observed for tetrapode $A$. We can also evaluate the transverse component of the dipole moment. This is found to be $\mu_{t}=0.45 \mathrm{D}$, again by assuming that in the vicinity of the isotropic phase $g \cong 1$. The dipole moment predicted by the DFT for simulated structure of a single monomer is found to be larger compared to the experimental value. Due to the specific arrangement of the branches, the overall dipoles of the monomers can compensate with each other and an additional cancellation may occur due to the relative orientation of the carbonyl dipoles in the mesogen.

The relaxation below the $N$-SmC phase transition temperature of $358 \mathrm{~K}$ shows quite interesting behavior [Fig. $6(\mathrm{a})]$ in terms of the dielectric relaxation strengths. In particular the dielectric strength of the low frequency mode shows soft modelike temperature dependence. On the other hand, the corresponding relaxation frequency [Fig. 6(b)] shows only a small anomaly. The second interesting feature appears at a temperature of $352 \mathrm{~K}$ seen in both Figs. 6(a) and 6(b). This is shown as a step in both the dielectric relaxation strengths and the relaxation frequencies. This anomaly also appears in IR data [16] but no transition was detected by the DSC signal around this temperature. This is quite an intriguing result and appears due to a possible occurrence of $\operatorname{Sm} A$ phase sandwiched in between the $N$ and $\operatorname{SmC}$ phases but its explanation awaits a detailed examination of this phase.

\section{Relaxation dynamics of the molecular relaxation processes}

The relaxation dynamics of the various modes is predicted by Eqs. (2a)-(2d) in a liquid crystalline nematic phase relative to their corresponding behavior in the isotropic phase. In order to analyze the relaxation frequency of the process it is convenient to use the Arrhenius equation $\tau=A^{\prime} \exp \left(E_{a} / k T\right)$ and extrapolate the frequency from the isotropic phase to the temperature range of the nematic phase. Here $A^{\prime}$ is a preexponential factor and $E_{a}$ is activation energy. For tetrapode $A$ we have found [Fig. 4(b)] that the low frequency process $\left(f_{1}\right)$ is retarded and the middle frequency processes $\left(f_{2}\right.$ and $f_{3}$ ) are accelerated with respect to the frequency $f_{0}$ in the isotropic phase taken as the reference. The frequency of the fourth mode does not appear to be so strongly influenced by the orientational order parameter.

The effect of retardation of the low frequency mode described by $\tau_{00}$ can be analyzed using Eq. (2a) in terms of the nematic pseudopotential barrier, $\sigma$, which depends on the order parameter. Coffey and Kalmykov [26,27] derived the relation between $\sigma$ and $S$. This can well be approximated as

$$
\sigma \approx \frac{3 S(5-\pi S)}{2\left(1-S^{2}\right)} .
$$

In order to reproduce the relaxation dynamics of the mode for tetrapode $A$ we used the M-S model [35] to describe the dependence of $S$ (as in Fig. 5) on temperature and then nematic potential barrier, $\sigma(T)$, was obtained using Eq. (7). Such a result was introduced into Eq. (2a) and the temperature dependence the frequency of mode 1 was obtained, see Fig. 4(b). This is found to be in reasonable agreement with the experiment. However, in order to obtain such a good fit, it was necessary to multiply $\sigma$ obtained from Eq. (7) by a factor of 1.13. This factor lies in between the predictions of Coffey-Kalmykov [26,27] and those of the Meier-Saupe [28] formula. This is not surprising in view of the fact that Eqs. (2a) and (7) are only approximations to the rather complicated expressions. The next relaxation process (mode 2) is characterized by the relaxation time $\tau_{10}$ and it is accelerated with respect to the isotropic phase. Its behavior was well replicated by using the same $S$ parameter as in Fig. 5, which was introduced into Eq. (2c), see Fig. 4(b). The next process (mode 3 ) is assigned to the relaxation time $\tau_{11}$ and its relative behavior should be described by Eq. (2d). The dependences of the calculated relaxation time relative to the isotropic phase are plotted as a function of the order parameter, $S$, in Fig. 8 for tetrapode $A$. By using Eq. (2d), we can evaluate the unknown behavior of the parameter, $\Delta$, which is a measure of the anisotropy of the rotational diffusion tensor, $D_{\|} / D_{\perp}$. We have found that this anisotropy increases from unity at the $T_{I-N}$ transition temperature to almost 3 in the nematic phase at room temperature. This is indeed a significant in- 


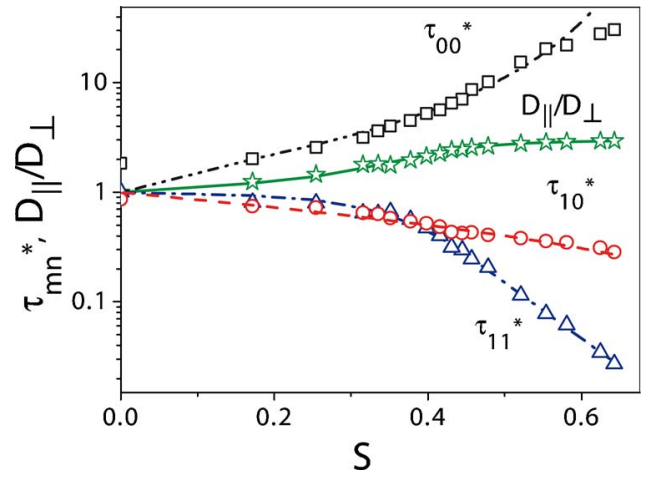

FIG. 8. (Color online) Plot of the reduced rotational relaxation times for tetrapode $A$, using the rotational diffusion model of Coffey and Kalmykov as a function of the order parameter. Reduced relaxation times $\tau_{00}{ }^{*}=\tau_{00} / \tau_{D}-\square, \tau_{10}{ }^{*}=\tau_{10} / \tau_{D}-\bigcirc, \tau_{11}{ }^{*}=\tau_{11} / \tau_{D}-\triangle$. The connecting lines are drawn from the predictions of the model. Anisotropy of the rotational diffusion coefficient, $D_{\|} / D_{\perp}-\star$.

crease in the anisotropy of the rotational diffusion tensor for such a system.

For tetrapode $B$ with symmetrical mesogen in the temperature range of the nematic phase it is not possible to evaluate $\tau_{00}$, since the relaxation process due to the end-overend rotation goes to frequencies below the window of the experiment. The low frequency process in the frequency range $10-100 \mathrm{~Hz}$ is assigned to mode 2 . This corresponds to the relaxation time $\tau_{10}$ and again is well reproduced by Eq. (2c), see Fig. 6(b). The next process in the kilohertz frequency range shows very similar temperature dependence. Most probably this process is due to the internal rotation (around the biphenyl para-axis) of one half of the monomeric unit, which is not attached to the spacer and assigned to mode 2int and is designated as $\tau_{10}$. The next relaxation peak, mode 3 , seems to be the rotation along the long molecular axis-also called the spinning motion and is described by the relaxation time $\tau_{11}$. For mode 3 , we are able to calculate the temperature dependence of the parameter $\Delta$ and the anisotropy of the rotational diffusion tensor, $D_{\|} / D_{\perp}$. This anisotropy increases from 1 at the transition temperature, $T_{I-N}$, to almost 3 at the transition temperature, $T_{N-S m C}$ similar to that for tetrapode $A$. The ratios of the various relaxation times as a function of the order parameter to the Debye time for the order parameter equal to zero, are plotted in Fig. 9. The Debye time at a given temperature for zero order parameter is evaluated by extrapolating the relaxation frequency from the isotropic phase to the given phase and the temperature, by assuming the same temperature dependence as in the isotropic phase. The results compare favorably with those predicted by the Coffey and Kalmykov model. The quality of modelling of relaxation frequencies is also seen in Fig. 6(b). The highest frequency process, mode 4 , arises most probably from the internal rotation of the smaller segments of the molecules and is not discussed further due to its rather weak temperature dependence.

The problem of the structural changes (with reference to the molecular shape) of the polymer has been studied by

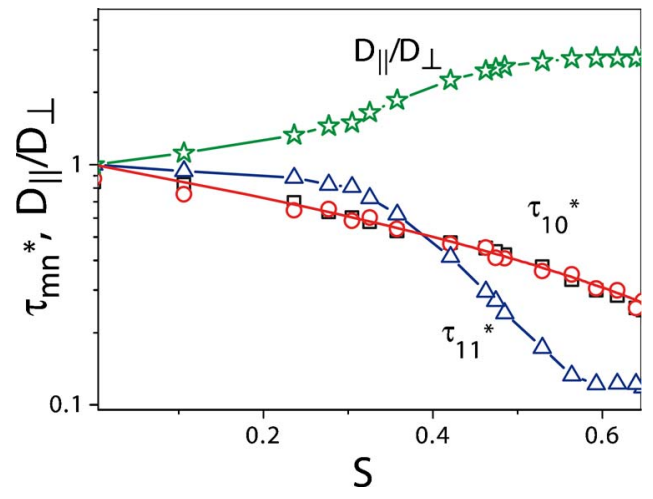

FIG. 9. (Color online) Plot of the reduced relaxation times for tetrapode $B$ using the rotational diffusion model of Coffey and Kalmykov as a function of the order parameter. Reduced relaxation times: $\tau_{10}{ }^{*}=\tau_{10} / \tau_{D}$ for mode $2-\square$, and for mode 2int- $\bigcirc$, $\tau_{11}{ }^{*}=\tau_{11} / \tau_{D}-\triangle$. The connecting lines are predictions of the model. Anisotropy of the rotational diffusion coefficient, $D_{\|} / D_{\perp}-\star$.

Pickett and Schweizer [36], their investigations were focused onto the nematic phase. They assume the existence of the two density correlation lengths in the anisotropic phase. Increasing the order parameter shrinks the invaded space of each chain, so that the neighboring chains overlap to a lesser extent. As a consequence of the anisotropy and because the monomeric branches adopt calamitic shapes, the rotation around the long molecular axis is expected to undergo much smaller friction when the molecular order increases on going from the isotropic to the nematic phase. On the other hand, the evaluated ratio $D_{\|} / D_{\perp}$ can be related to the molecular shape [37].

\section{CONCLUSIONS}

The dielectric relaxation spectra for each of the tetrapodes $A$ and $B$, has been resolved in terms of at least four relaxation processes. These tetrapodes are built up of asymmetric and symmetric monomeric units, respectively. The three molecular modes with increase in frequency are: the end-overend rotation, the precessional motion about the director, and the rotation about its own long molecular axis (also called the spinning motion). The relaxation rate of the first process is retarded whereas of the other two processes are accelerated in the nematic phase with respect to their relaxation rate in the isotropic phase. The changes are much more significant than for low molar mass LCs. The modes due to the internal rotation of the molecular segments are the high frequency mode (mode 4 ) and the intermediate frequency mode (mode 2int for tetrapode $B$ ). The orientational order parameters have been determined using the dielectric strength of the low frequency modes. The results agree with those obtained by the method using IR spectroscopy. The temperature dependences of the order parameter are well described by the Maier-Saupe model. The critical exponents are found to be slightly lower than the value for the so-called tricritical point. The dynamics of the relaxation modes are quantitatively analyzed in terms of the microscopic model of Coffey 
and Kalmykov for the rotational diffusion of the molecules in the nematic potential. The temperature dependence of the relaxation rate is well predicted by the model. The anisotropies of the rotational diffusion coefficients have been evaluated and these gradually increase with a decrease in temperature up to the factor of 3 in the nematic phase relative to that in the isotropic phase. Dielectric anisotropic properties and the dynamical behavior are controlled mostly by the orientational order and the properties of the monomer. The results lead to the conclusion that the monomeric units are somewhat decoupled from the core of the tetrapodes.

\section{ACKNOWLEDGMENTS}

This work was supported by the EU network project on liquid crystalline dendrimers HPRN CT-2000-00016 under which K.M. was the postdoctoral Fellow in Trinity College Dublin where the work was carried out. The appointment of A.K. in Dublin was funded by SFI (02/IN.1/I031), and the SFI overhead investment plan 2005, to which we acknowledge. The authors (A.K. and K.M.) also acknowledge the support of the Committee for Scientific Research (KBN): Grant No. 2P03B 07025.
[1] S. A. Ponomarenko, E. A. Rebrov, A. Y. Bobrovsky, A. M. Muzafarov, and V. P. Shibaev, Liq. Cryst. 21, 1 (1996).

[2] M. W. P. L. Baars, S. H. M. Söntjens, H. M. Fischer, H. W. J. Peerlings, and E. W. Meijer, Chem.-Eur. J. 4, 2456 (1998).

[3] K. Lorenz, D. Hölter, B. Stühn, R. Mühlhaupt, and H. Frey, Adv. Mater. (Weinheim, Ger.) 8, 414 (1996).

[4] R. M. Richardson, S. A. Ponomarenko, N. I. Boiko, and V. P. Shibaev, Liq. Cryst. 26, 1 (1999).

[5] V. Percec, P. W. Chu, and M. Kawasumi, Macromolecules 27, 4441 (1994).

[6] J. H. Cameron, A. Facher, G. Lattermann, and S. Diele, Adv. Mater. (Weinheim, Ger.) 9, 347 (1997).

[7] V. Percec, W. D. Cho, and G. Ungar, J. Am. Chem. Soc. 119, 1539 (2000).

[8] X. Zeng, G. Ungar, L. Yongsong, V. Percec, A. E. Dulcey, and J. K. Hobbs, Nature (London) 428, 157 (2004).

[9] J. M. Rueff, J. Barbera, B. Donnio, D. Guillon, M. Marcus, and J. L. Serrano, Macromolecules 36, 8368 (2003).

[10] J. Barbera, B. Donnio, R. Gimenez, D. Guillon, M. Marcus, A. Omenat, and J. L. Serrano, J. Mater. Chem. 11, 2808 (2001).

[11] G. H. Mehl and J. W. Goodby, Chem. Ber. 129, 521 (1996).

[12] G. H. Mehl and J. W. Goodby, Chem. Commun. (Cambridge), 13 (1999).

[13] R. Elsässer, G. H. Mehl, J. W. Goodby, and M. Veith, Angewandte Chemie, Angew. Chem., Int. Ed. 40, 2688 (2001).

[14] L. Tajber, A. Kocot, J. K. Vij, K. Merkel, J. Zalewska-Rejdak, G. H. Mehl, R. Eläßer, J. W. Goodby, and M. Veith, Macromolecules 35, 8601 (2002).

[15] T. Meyer and G. H. Mehl (unpublished).

[16] K. Merkel, A. Kocot, J. K. Vij, G. H. Mehl, and T. Meyer, J. Chem. Phys. 121, 5012 (2004).

[17] P. G. de Gennes and J. Prost, The Physics of Liquid Crystals, 2nd ed. (Oxford Science, Oxford, 1993).

[18] D. Dunmur and K. Toriyama, in Handbook of Liquid Crystals, edited by D. Demus, J. W. Goodby, G. W. Gray, H. W. Spiess, and V. Vill (Wiley-VCH, Weinham, 1998), Vol. 1, Chap. VII.1, p. 189.

[19] D. A. Dunmur and K. Toriyama, in Handbook of Liquid Crys- tals, edited by D. Demus, J. W. Goodby, G. W. Gray, H. W. Spiess, and V. Vill (Wiley-VCH, Weinham, 1998), Vol. 1, Chap. VII.4, p. 231.

[20] D. A. Dunmur, G. R. Luckhurst, M. R. de la Fuente, S. Diez, and M. A. Perez Jubindo, J. Chem. Phys. 115, 8681 (2001).

[21] S. Diez, D. A. Dunmur, M. R. de la Fuente, P. K. Karahaliu, G. H. Mehl, T. Meyer, M. A. Perez Jubindo, and D. J. Photinos, Liq. Cryst. 30, 1021 (2003).

[22] C. M. Haws, M. G. Clark, and G. S. Attard, Side Chain Liquid Crystal Polymers (Blackie, Glasgow, 1989), p. 196, edited by C. B. McArdle.

[23] W. Maier and G. Meier, Z. Naturforsch. A 16A, 262 (1961).

[24] K. Merkel, A. Kocot, J. K. Vij, R. Korlacki, G. H. Mehl, and T. Meyer, Phys. Rev. Lett. 93, 237801 (2004).

[25] P. L. Nordio, G. Rigatti, and U. Segre, Mol. Phys. 25, 129 (1973).

[26] W. T. Coffey and Yu. P. Kalmykov, Advances in Liquid Crystals, edited by J. K. Vij (Wiley, New York, 2000); Adv. Chem. Phys. 113, 487 (2000).

[27] W. T. Coffey, Yu. P. Kalmykov, and J. T. Waldron, Liq. Cryst. 18, 677 (1995).

[28] G. Meier and A. Saupe, Mol. Cryst. 1, 515 (1966).

[29] M. Stocchero, A. Ferrarini, G. J. Moro, D. A. Dunmur, and G. R. Luckhurst, J. Chem. Phys. 121, 8079 (2004).

[30] S. Havriliak, Jr. and S. Negami, J. Polym. Sci., Part C: Polym. Symp. 14, 99 (1966).

[31] A. Perico, R. Pratolongo, K. F. Freed, R. W. Pastor, and A. Szabo, J. Chem. Phys. 98, 564 (1993).

[32] S. J. Picken, L. Noirez, and G. R. Luckhurst, J. Chem. Phys. 109, 7612 (1998).

[33] W. Maier and A. Saupe, Z. Naturforsch. A 14A, 882 (1959).

[34] I. Chirtoc, M. Chirtoc, C. Glorieux, and J. Thoen, Liq. Cryst. 31, 229 (2004).

[35] W. Maier and A. Saupe, Z. Naturforsch. A 15A, 287 (1960).

[36] G. T. Pickett and K. S. Schweizer, J. Chem. Phys. 112, 4869 (2000).

[37] C. Hu and R. Zwanzig, J. Chem. Phys. 60, 4353 (1974). 